\title{
AVALIAÇÃO ENERGÉTICA DE UM SISTEMA DE ESGOTAMENTO SANITÁRIO: O CASO DE FEIRA DE SANTANA-BA
}

Samuel Sipert ${ }^{1}$; Eduardo Cohim²; Renato Campos ${ }^{2}$, Hamilton da Silva Neto ${ }^{2}$

1 Universidade Estadual de Feira de Santana; Feira de Santana/BA; ssipert@hotmail.com

${ }^{2}$ Universidade Estadual de Feira de Santana; Feira de Santana/BA

Resumo: Com o objetivo de avaliar a eficiência energética para um sistema municipal de esgotamento sanitário através de uma ACV, foram coletados dados do Sistema de Esgotamento Sanitário de Feira de Santana (SES-FSA) para criação de um Inventário de Ciclo de Vida (ICV). O impacto do ciclo de vida foi avaliado com base no método Cumulative Energy Demand (CED). Cadeias de produção e transporte para tubulação substituída no sistema e transporte de lodo de esgoto para aterro sanitário também foram consideradas. A demanda energética acumulada para o SES-FSA foi de 0,76 $\mathrm{kWh} \cdot \mathrm{m}^{-3}$ de esgoto tratado, com maior encargo proveniente da etapa de tratamento, representando $73 \%$ do total.

Palavras-Chave: ACV; esgoto; sustentabilidade

\section{ENERGY ASSESSMENT OF A MUNICIPAL SEWAGE SYSTEM: THE CASE OF FEIRA DE SANTANA-BA}

\begin{abstract}
In order to evaluate the energy efficiency for a municipal sewage system through a LCA, data were collected from the Feira de Santana's Municipal Sewage System (SES-FSA) to create a Life Cycle Inventory (LCI). Life cycle impact was assessed based on the Cumulative Energy Demand (CED) method. Production and transport chains for replaced pipe in the system and transportation of sewage sludge to landfill were also considered. The accumulated energy demand for the SES-FSA was $0.76 \mathrm{kWh} \cdot \mathrm{m}^{-3}$ of treated sewage, with the largest burden coming from the treatment stage, representing $73 \%$ of the total.
\end{abstract}

Keywords: LCA; wastewater; sustainability 
1. INTRODUÇÃOA interconexão entre água e energia tem se tornado cada vez mais relevante à medida em que crescem as pressões causadas pelo aumento populacional e dos efeitos das mudanças climáticas sobre os recursos hídricos e seus regimes de vazão [1]. Enquanto energia é necessária para as etapas de transporte e tratamento de água e esgoto, água é necessária, diretamente ou indiretamente, para a produção de energia, influenciando assim seu custo de geração. Para o Brasil, essa relação intrínseca representa desafios ainda maiores, tendo em vista que $68,1 \%$ da matriz elétrica do país é dependente de fontes hidráulicas [2].

Além disso, de acordo com o Balanço Energético Nacional (BEN), o Brasil consumiu em 2015, 522,8 TWh de energia elétrica [3], dos quais 12,2 TWh foram destinados a serviços de saneamento [4], o que representa $2,3 \%$ de toda energia consumida no país.

Dentro desse contexto, e considerando a crescente demanda de água e energia, surge a necessidade de adoção de uma abordagem mais sistêmica para o gerenciamento da água e dos esgotos urbanos, com práticas que permitam reaproveitamento e uma maior eficiência de uso dos recursos, além de adaptabilidade frente ao desenvolvimento das cidades.

Historicamente, o principal objetivo dos sistemas de esgotamento sempre foi o de garantir boas condições de saúde à população por meio de implantação de coleta centralizada e grandes estruturas para tratamento. Nos dias atuais, com o crescimento da consciência sustentável, o esgoto já é visto como fonte alternativa de água e energia, bem como de recuperação de nutrientes [1]. Portanto, novas tecnologias que não apenas garantam níveis de qualidade satisfatórios a custos aceitáveis, mas também integrem as novas preocupações de eficiência energética e sustentabilidade ambiental em suas operações devem ser estudadas.

Métodos como a avaliação de ciclo de vida (ACV) podem ser utilizados nesse sentido, proporcionando uma visão mais abrangente dos aspectos ambientais e energéticos associados a um serviço ou produto, sendo utilizado mundialmente para avaliar a sustentabilidade de sistemas urbanos de água e esgoto, propondo cenários mais eficientes para o seu gerenciamento [5,6,7].

A fim de contribuir para a melhor gestão energética em sistemas de esgotamento, tornando-os mais sustentáveis, o presente trabalho teve como objetivo quantificar, através de uma Avaliação de Ciclo de Vida (ACV), a demanda acumulada de energia para a fase de operação do sistema de esgotamento sanitário de Feira de Santana - BA (SES-FSA), permitindo uma visão mais ampla para a continuação deste trabalho na criação de cenários alternativos.

\section{METODOLOGIA}

O método de análise utilizado neste trabalho foi a Avaliação de Ciclo de Vida (ACV), baseada nas normas da Associação Brasileira de Normas Técnicas (ABNT) NBR ISO 14040 [8] e 14044 [9]. A ACV foi realizada em quatro fases distintas: 
definição do objetivo e escopo, análise de inventário, avaliação do impacto do ciclo de vida e interpretação.

A fim de contabilizar a energia primária utilizada durante o ciclo de vida do produto, foi utilizado o método Cumulative Energy Demand (CED).

\subsection{Definição de escopo}

A demanda acumulada de energia para o SES-FSA foi quantificada através de dados de operação das etapas de coleta e tratamento de esgoto para o ano-base de 2016. A abordagem utilizada para a modelagem do sistema foi a ACV atribucional, tendo-se em vista o uso de históricos e dados mensuráveis para a atribuição de potenciais impactos ambientais para o sistema estudado [7,10].

O sistema de coleta é composto por 27 Estações Elevatórias de Esgoto (EEE) espalhadas por diversos pontos cidade, com uma malha de tubulação de 596850 metros de extensão, composta principalmente por PVC rígido. As bombas utilizadas nas EEE operam em baixa tensão (110-440V).

Por sua vez, o sistema de tratamento é composto por 3 Estações de Tratamento de Esgoto (ETE) principais que são responsáveis por $80 \%$ do tratamento de volume total (Jacuípe I, Jacuípe II e Subaé). Estas operam à média tensão (138kV), atendendo a 146563 economias. O restante do volume é tratado por 25 ETEs menores, que operam a baixa tensão, implantadas em condomínios e empreendimentos imobiliários, atendendo cerca de 24134 economias.

\subsection{Unidade funcional}

A unidade funcional adotada foi de $1 \mathrm{~m}^{3}$ de esgoto tratado.

\subsection{Fronteiras do sistema}

A fronteira do presente trabalho foi classificada como "berço ao portão", e se limitou à fase de operação do sistema, incluindo a coleta do esgoto residencial pelo sistema coletor, o transporte até as estações de tratamento, e a descarga de esgoto tratado aos corpos receptores. Foram consideradas também as cadeias de produção e transporte de materiais utilizados durante a operação.

Os encargos energéticos provenientes da fase de construção e implantação do sistema não foram incluídos na avaliação tendo-se em vista a falta de informações sobre os quantitativos de materiais e energia utilizados em obra. Além disso, segundo a literatura, o impacto da fase de construção geralmente não ultrapassa $5 \%$ dos impactos causados pela fase de operação durante o ciclo de vida [7].

As fronteiras utilizadas neste trabalho podem ser vistas na Figura 1. 
Figura 1. Fronteiras do sistema

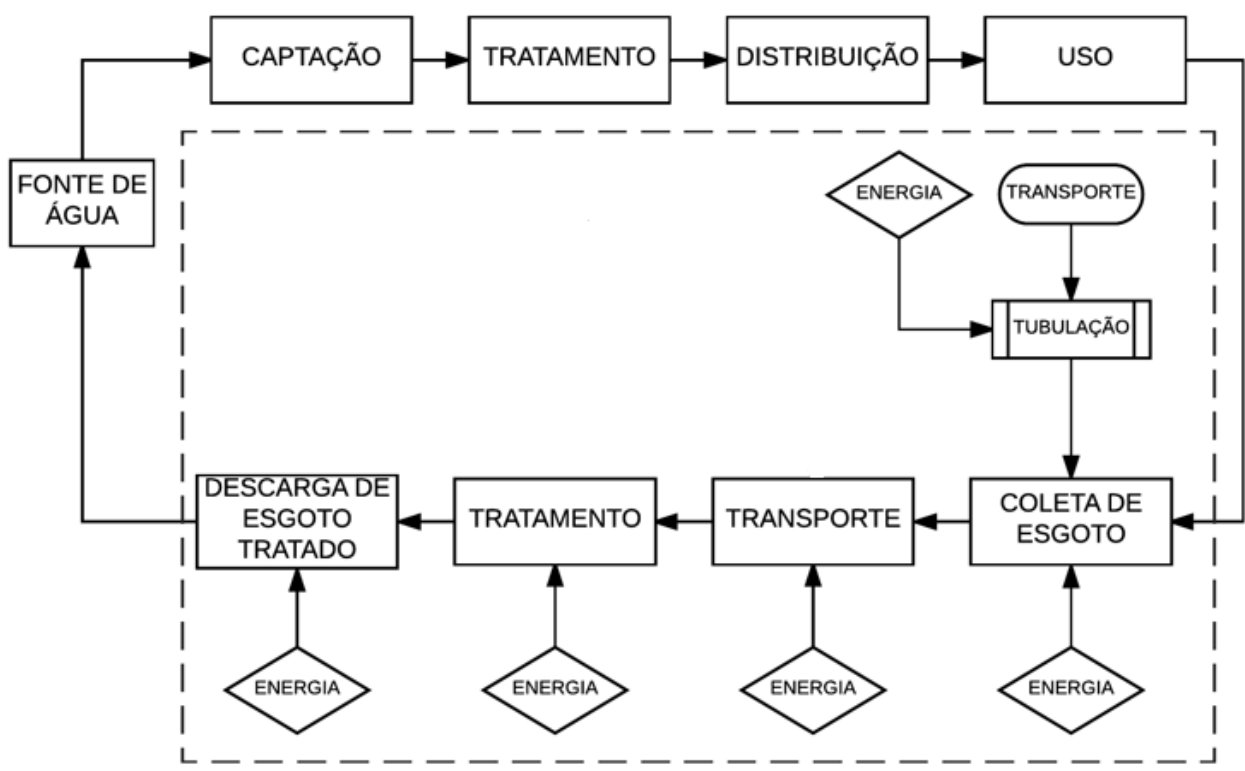

\subsection{Inventário de ciclo de vida}

Os dados de volume total de esgoto tratado para o ano de 2016 foram estimados através da Equação 1, com base no número de economias atendidas, dado este que foi cedido pela Empresa Baiana de Águas e Saneamento (EMBASA).

$$
V_{\text {esg }}=\left(N_{\text {econ }} \times T_{\text {ocup }} \times q_{\text {per capita }} \times K_{\text {águalesgoto }} \times 365\right) / 1000
$$

VESG é o volume de esgoto tratado, em m³ ${ }^{3}$ NECON é o número de economias atendidas; TOCUP é taxa de ocupação residencial, em habitantes por residência; qPER CAPITA é o consumo per capita em $\mathrm{L} \cdot \mathrm{hab}^{-1} \cdot \mathrm{dia}^{-1}$; e KÁGUA/ESGOTO é a taxa de conversão de água potável consumida para esgoto produzido.

Assumiu-se uma taxa de ocupação média de 3,7 habitantes por residência, um consumo per capita igual a $120 \mathrm{~L} \cdot \mathrm{hab}^{-1} \cdot \mathrm{dia}^{-1}[11]$ e uma taxa de conversão de água consumida para esgoto de 0,8 . O volume final de esgoto tratado estimado para 2016 foi de $14791721,5 \mathrm{~m}^{3}$.

Os dados para o consumo de energia elétrica das EEEs e ETEs do sistema foram fornecidos pela EMBASA para o período de janeiro a dezembro de 2016. Os fatores de conversão de energia primária para energia elétrica, para baixa e média tensão, foram retirados da base de dados do Ecolnvent versão 3.3, considerando a matriz energética brasileira.

Para o cálculo dos insumos e materiais utilizados durante o período, admitiu-se uma taxa anual de substituição da tubulação de $2 \%$ devido à danos na rede de coleta. Assumiu-se uma tubulação composta por PVC rígido, com um diâmetro médio de 200 $\mathrm{mm}$, e uma densidade de $4,27 \mathrm{~kg} \cdot \mathrm{m}^{-1}$. O consumo energético para o transporte da 
tubulação foi calculado através do momento de transporte, adotando-se uma distância da fábrica de tubulação até a sede do revendedor de 460 quilômetros. Todos as variáveis utilizadas para o cálculo estão dispostas na Tabela 1. Os dados secundários para a quantificação da energia embutida nos insumos estão dispostos na Tabela 2.

Tabela 1. Cálculo da massa dos tubos substituídos

\begin{tabular}{|l|c|c|}
\hline \multicolumn{1}{|c|}{ Tubulação } & Quantidade & Unidade \\
\hline Comprimento da tubulação & 596850,00 & $\mathrm{~m}$ \\
\hline Massa por metro & 4,27 & $\mathrm{~kg} \cdot \mathrm{m}^{-1}$ \\
\hline Taxa de substituição & $2 \%$ & - \\
\hline Comprimento substituído & 11937,00 & $\mathrm{~m}$ \\
\hline Massa total & 50970,99 & $\mathrm{~kg}$ \\
\hline
\end{tabular}

Tabela 2. Dados dos insumos do Sistema de Esgotamento Sanitário de Feira de Santana

\begin{tabular}{|l|c|c|c|}
\hline \multicolumn{1}{|c|}{ Produtos } & $\begin{array}{c}\text { Energia } \\
\text { embutida }\end{array}$ & Unidade & $\begin{array}{c}\text { Insumo do Ecoinvent } \\
\text { Eletricidade baixa voltagem }\end{array}$ \\
\hline Eletricidade média voltagem & 1,86 & $\mathrm{kWh} \cdot \mathrm{kWh}^{-1}$ & $\begin{array}{c}\text { Electricity, low voltage } \\
\text { (BR) }\end{array}$ \\
\hline Tubos substituídos & 27,39 & $\mathrm{kWh} \cdot \mathrm{kWh}^{-1}$ & $\begin{array}{c}\text { Electricity, medium } \\
\text { voltage (BR) }\end{array}$ \\
\hline Transporte & 1,01 & $\mathrm{kWh} \cdot \mathrm{kg}^{-1}$ & $\begin{array}{c}\mathrm{PVC} \text { pipe, DN 200, } \\
\text { SDR 41 (RoW) }\end{array}$ \\
\hline Massa total & 50970,99 & $\mathrm{~kg}$ & $\begin{array}{c}\text { Transport, truck 10- } \\
\text { 20t, EURO5 }\end{array}$ \\
\hline
\end{tabular}

\section{RESULTADOS E DISCUSSÃO}

A Tabela 3 apresenta o resultado do inventário e as contribuições do CED para cada etapa do ciclo de vida do SES - FSA. Os resultados foram agregados por etapas para identificação dos processos com maior encargo energético.

De acordo com os resultados, o sistema demanda o total de 0,76 kWh de energia acumulada para cada $\mathrm{m}^{3}$ de esgoto tratado. Esse resultado está dentro da faixa de consumo energético para sistemas de esgotamento sanitário de 0,29-1,22 $\mathrm{kWh} \cdot \mathrm{m}^{-3}$ estimado por Meda et al. [12]. Considerando as etapas de coleta e tratamento, $88 \%$ do valor de demanda acumulada de energia está relacionado ao consumo de energia elétrica, corroborando resultados encontrados por Guanais et al. [7] e Lemos et al. [13], que avaliaram sistemas de água e esgoto e atribuíram os maiores impactos ao consumo de eletricidade. 


\section{\begin{tabular}{l|l} 
CIRCULAR ECONOMY & ECONOMIA CIRCULAR
\end{tabular}}

Tabela 3. Inventário de Ciclo de Vida para 14.791.721,5 $\mathrm{m}^{3}$ de esgoto tratado

\begin{tabular}{|c|c|c|c|c|}
\hline Etapa & Quantidade & Unidade & $\begin{array}{l}\text { Demanda de energia } \\
\text { acumulada (kWh) }\end{array}$ & ${ }^{*} \mathrm{kWh} \cdot \mathrm{m}^{-3}$ \\
\hline \multicolumn{5}{|l|}{ Coleta } \\
\hline Eletricidade & 769502,98 & kWh & 1654431,41 & 0,11 \\
\hline Tubos & 50970,99 & $\mathrm{~kg}$ & 1396095,42 & 0,09 \\
\hline Transporte & 23446,66 & tkm & 10550,99 & 0,00 \\
\hline Subtotal & & & 3061077,82 & 0,21 \\
\hline \multicolumn{5}{|l|}{ Tratamento } \\
\hline \multicolumn{5}{|c|}{ Subaé/Jacuípe I/Jacuípe II } \\
\hline Eletricidade & 3789710,46 & $\mathrm{kWh}$ & 7041282,03 & 0,48 \\
\hline \multicolumn{5}{|l|}{ ETEs menores } \\
\hline Eletricidade & 540739,66 & kWh & 1162590,27 & 0,08 \\
\hline Subtotal & & & 8203873,11 & 0,55 \\
\hline \multicolumn{4}{|c|}{ TOTAL } & 0,76 \\
\hline
\end{tabular}

A etapa de tratamento de esgoto representou o maior encargo, com 0,55 $\mathrm{kWh} \cdot \mathrm{m}^{-3}$ (73\% do total). A etapa de coleta demandou $0,21 \mathrm{kWh} \cdot \mathrm{m}^{-3}$, e é importante notar o impacto energético causado pela produção da tubulação substituída, que representou quase a metade desta demanda.

A Tabela 4 apresenta um comparativo entre os resultados encontrados neste estudo, com outros autores que também avaliaram o gasto energético de sistemas de esgotamento sanitário ao redor do mundo. É possível notar que os valores encontrados estão em conformidade com valores encontrados na literatura, sendo necessária uma avaliação mais detalhada para levantar os fatores que influenciam nessa questão.

Tabela 4. Gasto energético para sistemas urbanos de esgotamento sanitário $\left(\mathrm{kWh} \cdot \mathrm{m}^{-3}\right)$

\begin{tabular}{|c|c|c|}
\hline Fonte & Coleta & Tratamento \\
\hline Presente estudo & 0,21 & 0,55 \\
\hline$[5]$ & 0,00 & 1,09 \\
\hline$[13]$ & 0,21 & 0,87 \\
\hline$[14]$ & 0,04 & 0,17 \\
\hline$[15]$ & 0,06 & 0,75 \\
\hline$[16]$ & 0,14 & 0,44 \\
\hline
\end{tabular}




\section{CONCLUSÃO}

Através do método CED, é possível concluir que os maiores encargos energéticos do SES-FSA são provenientes do consumo de energia elétrica. A etapa mais representativa é o tratamento, com $72 \%$ da demanda acumulada de energia total. Com base nesses dados, os cenários alternativos de reaproveitamento energético a serem estudados no futuro, devem se concentrar prioritariamente nas estações de tratamento, em detrimento de soluções no sistema de coleta.

\section{REFERÊNCIAS}

1 LAZAROVA, V.; CHOO, K.-H.; CORNEL, P. Water-energy interactions in water reuse. IWA publishing. ISBN 184339541X, 2012.

${ }^{2}$ BRASIL. Balanço Energético Nacional - BEN. Ano-base: 2016. Rio de Janeiro: MMEEPE. 296p, 2017.

${ }^{3}$ BRASIL. Balanço Energético Nacional - BEN. Ano-base: 2015. Rio de Janeiro: MMEEPE. 294p, 2016.

${ }^{4}$ BRASIL. Sistema Nacional de Informações sobre Saneamento: diagnóstico dos serviços de água e esgotos. Brasília: SNSA/MCIDADES. 212p, 2015.

${ }^{5}$ AMORES, M. J.; MENESES, M.; PASQUALINO, J.; ANTÓN, A.; CASTELLS, F. Environmental assessment of urban water cycle on Mediterranean conditions by LCA approach. Journal of cleaner production, V.43, pp. 84-92. ISSN 0959-6526, 2013.

${ }^{6}$ LOUBET, P.; ROUX, P.; LOISEAU, E.; BELLON-MAUREL, V. Life cycle assessments of urban water systems: A comparative analysis of selected peer-reviewed literature. Water research, V.67, pp. 187-202. ISSN 0043-1354, 2014.

7 GUANAIS, A. L. R.; COHIM, E. B.; MEDEIROS, D. L. Energy evaluation of an integrated water supply system. Engenharia Sanitaria e Ambiental. ISSN 14134152, 2017.

${ }^{8}$ ASSOCIAÇÃO BRASILEIRA DE NORMAS TÉCNICAS - ABNT. NBR ISO 14040: Gestão ambiental - Avaliação do ciclo de vida - Princípios e estrutura. Rio de Janeiro, 2009a.

${ }^{9}$ ASSOCIAÇÃO BRASILEIRA DE NORMAS TÉCNICAS - ABNT. NBR ISO 14044: Avaliação do Ciclo de Vida: Requisitos e Orientações. Rio de Janeiro, 2009b.

${ }^{10}$ EUROPEAN COMMISSION. ILCD Handbook: international reference life cycle data system: general guide for life cycle assessment. Luxemburgo: Publications Office of the European Union. 417p, 2010.

11 EMPRESA BAIANA DE ÁGUAS E SANEAMENTO - EMBASA. Relatório de controle operacional de sistema de esgotamento sanitário (COPAE). 2016. 
${ }^{12}$ MEDA, A.; LENSCH, D.; SCHAUM, C.; CORNEL, P. Energy and water: relations and recovery potential. Water-energy interactions of water reuse. IWA Publishing, London, pp. 21-35, 2012.

${ }^{13}$ LEMOS, D.; DIAS, A.C.; GABARRELL, X.; ARROJA, L. Environmental assessment of an urban water system. Journal of Cleaner Production, V.54, pp. 157-165, 2013. ${ }^{14}$ BARJOVEANU, G.; COMANDARU, I.M.; RODRIGUEZ-GARCIA, G.; HOSPIDO, A.; TEODOSIU, C. (2013) Evaluation of water services system through LCA. A case study for lasi City, Romania. The International Journal of Life Cycle Assessment, V.19, pp. 449-462.

${ }^{15}$ VENKATESH, G.; BRATTEBO, H. Energy consumption, costs and environmental impacts for urban water cycle services: Case study of Oslo (Norway). Energy, V. 36, pp. 792-800, 2011. 